\title{
US to take temperature of mercury threat
}

\section{Mark Schrope}

In the United States alone this year, some 60,000 babies may be born with neurological damage caused by mercury poisoning of their mothers.

Scientists believe that much of the mercury enters the food chain through seafood whose habitat has been polluted by emissions from power stations burning mercury-rich coal. But it would be expensive - and therefore controversial - to regulate such emissions.

However, as one of several parting shots, President Bill Clinton's Environmental Protection Agency (EPA) last month laid out plans to implement such regulation by 2004 - and a research strategy to back it up.

Jonathan Herrmann, assistant director of the EPA's National Risk Management Research Laboratory in Cincinnati, Ohio, and a co-leader of the team that devised the strategy, says that the nature of the regulations will depend on the research. The plan is to explore the feasibility of different approaches to mercury removal, the distribution of emissions, and their effects on human health.

Some mercury is already removed from power-plant emissions along with other pollutants. The research will investigate by how much mercury emissions can be reduced using existing and emerging technologies, the relative cost of reductions, how much of the mercury in the environment can be attributed to power plants, and the probable effect of regulation on environmental mercury levels.

The answers will help the agency to determine not only how much mercury the power plants should be required to remove, but how much removal is economically reasonable. Currently, the EPA thinks regulation will cost

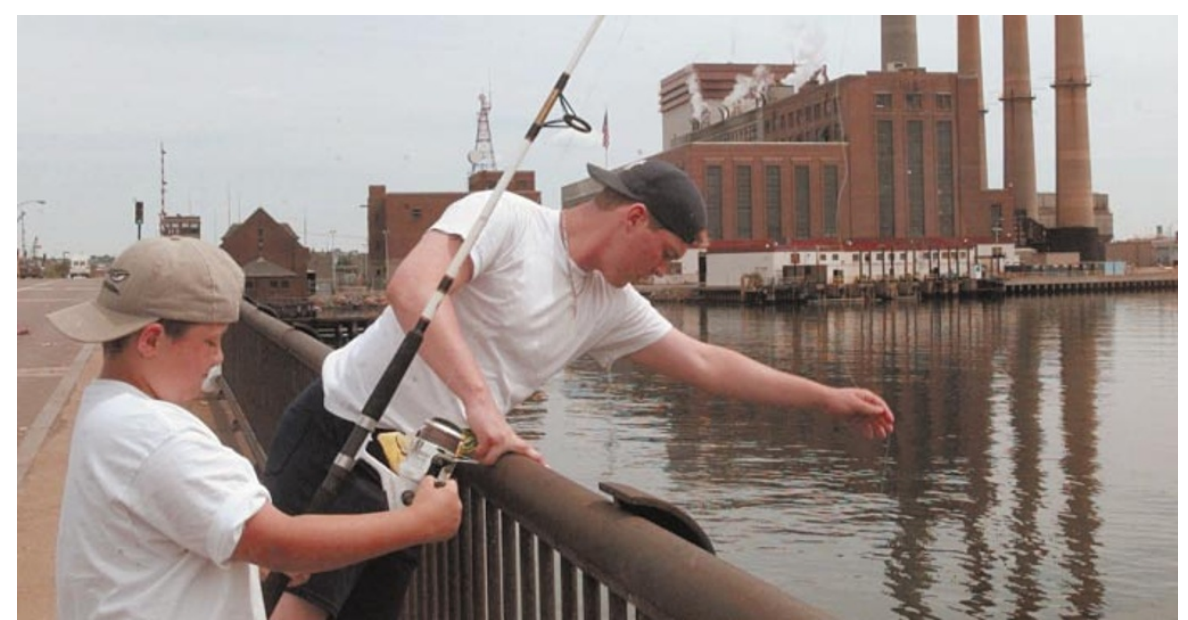

Throw it back: mercury emitted by power plants enters fish, and then the people that eat them.

between one billion and two billion dollars to implement, whereas industry estimates range from two billion to six billion dollars.

"It seems like a well-balanced strategy and I support wholeheartedly the work that they're doing," says Leonard Levin, a manager at the Electric Power Research Institute, based in Palo Alto, California. Levin, who was one of the strategy's outside reviewers, says it should provide a framework for establishing mercury regulations.

Mercury is released from power plants and waste incinerators in an inorganic form. But in water, microbes convert it into methylmercury, which accumulates in fish and shellfish. When the fish are eaten, methylmercury accumulates in the brain, where it reverts back to an inorganic form.

A National Academy of Science (NAS) study last year estimated that the mercury problem was substantial enough to influence the total number of children who struggle in school and require special help.

The NAS report, which contains the estimate that 60,000 mothers could be affected each year, also recommended research to understand the extent of mercury's effects on humans. For instance, there is some evidence that mercury causes cardiovascular problems in children.

Thomas Burke, an epidemiologist at Johns Hopkins University in Baltimore, and a lead author of the NAS study, says that, despite gaps in understanding, increased regulation is warranted.

"There is nothing to indicate that mercury risks are less than we thought," he says. "I think it is very appropriate to move forward aggressively to limit mercury in every way possible."

\section{Hunt for Earth-like planets edges towards launch pad}

Tony Reichhardt, Washington

A space telescope that would search for Earth-sized planets orbiting other stars is among three mission concepts picked by NASA last week to compete for a launch slot in 2005 or 2006.

The Kepler mission and two other proposals - Dawn to orbit the asteroids Vesta and Ceres, and INSIDE Jupiter to investigate the giant planet's interior by studying its magnetic and gravitational fields - were chosen as finalists from 26 ideas submitted to the agency's Discovery programme in August. Each team will receive $\$ 450,000$ to define its concept further, and a winner will be selected late this year. All three missions are priced at just under $\mathbf{\$ 3 0 0}$ million.

The choice of Kepler as a finalist is a vindication of sorts for principal investigator William Borucki of NASA's Ames Research Center in California. He has long proposed a novel and controversial method of searching for Earth-sized planets.

Placed into orbit around the Sun, and equipped with a 0.95 -metre telescope and ultra-sensitive charge-coupled device (CCD) detectors, Kepler would fix its gaze on 100,000 stars simultaneously, looking for extremely small dips in their light output. An Earth-sized planet passing in front of a Sun-like star would eclipse its light by only a few parts in 100,000.

But that would be enough to betray the planet's existence, says Borucki, if the pattern is regular. If Earth-sized planets are common, Kepler could detect more than 500 during its four-year lifetime.

^ ๑ 2001 Macmillan Magazines Ltd
Borucki has had trouble convincing NASA review committees that a spacecraft can achieve the sensitivity needed to register such minute changes in light. But he has since successfully tested his system in the laboratory, even when introducing the types of 'noise' that might be expected in space.

Planet hunters have found dozens of Jupiter-sized objects, but nothing like Earth. Borucki and his colleagues claim that their technique is the only one that can find Earthsized planets in the zone near a star where water would remain liquid at the surface.

But Kepler would not be the first such instrument to fly. The French-led Corot mission is scheduled to launch in late 2004, and will use a CCD array to look for transiting extrasolar planets. http://discovery.nasa.gov/news.html 